\title{
Chronic inflammatory demyelinating polyneuropathy-like neuropathy as an initial presentation of Crohn's disease
}

\author{
Suji Kim ${ }^{1+}$, Seok-Jae Kang ${ }^{2 \dagger}$, Ki-Wook Oh${ }^{2}$, Byung Kyu Ahn ${ }^{3}$, Hang Lak Lee ${ }^{4}$, Dong Soo Han ${ }^{4}$, Kiseok Jang ${ }^{5}$
} and Young Seo Kim²*

\begin{abstract}
Background: Chronic inflammatory demyelinating polyneuropathy (CIDP) is a rare complication of Crohn's disease (CD), and it is uncertain whether it is associated with CD itself or with its treatment. We describe a case of CIDP-like neuropathy as an initial symptom of CD. The neurologic symptoms of the patient which responded partially to intravenous immunoglobulin (IVIG) recovered after resection of the appendiceal CD.

Case presentation: A 17-year-old male had experienced three separate attacks of motor weakness and paresthesia of all four extremities over a period of 7 months. The electrophysiologic findings revealed a demyelinating sensory-motor polyneuropathy which was compatible with CIDP. However, repeated intravenous IVIG $(2 \mathrm{~g} / \mathrm{kg})$ treatment gave only a partial response. Four days after the last discharge, he was diagnosed as appendiceal CD after surgical resection of a periappendiceal abscess. His neurologic symptoms and electrophysiologic findings recovered without any maintenance therapy.

Conclusions: CIDP-like neuropathy can be an initial presentation of CD, and recovery of the CIDP symptoms may result from resection of the $C D$. Clinicians should be aware of the possibility of $C D$ in patients with intractable CIDP symptoms.
\end{abstract}

Keywords: Chronic inflammatory demyelinating polyneuropathy, Crohn's disease

\section{Background}

Crohn's disease $(C D)$ is a relapsing, transmural inflammatory disease of the gastrointestinal mucosa that can affect the entire gastrointestinal tract form mouth to anus [1]. It is considered a systemic disease because extraintestinal manifestations such as uveitis, arthritis, pleuritis, myocarditis, primary sclerosing cholangitis, pancreatitis, ankylosing spondylitis and tendinitis can develop [1]. Neurologic manifestations can also occur, the most common being myelopathy, arterial stroke, myopathy, multiple sclerosis and epilepsy [2-4]. Peripheral neuropathy is also reported to be a neurologic complication, and it can be associated with immune-mediated inflammation, micronutrient deficiencies (e.g., vitamin B12,

\footnotetext{
* Correspondence: aescula@hanmail.net

${ }^{\dagger}$ Equal contributors

${ }^{2}$ Department of Neurology, College of Medicine, Hanyang University,

17 Haengdang-dong, Seongdong-gu, Seoul 133-792, Republic of Korea Full list of author information is available at the end of the article
}

vitamin $\mathrm{D}$, copper) and iatrogenic causes (e.g., metronidazole, TNF- $\alpha$ antagonists) [3,5-8]. A few cases of chronic inflammatory demyelinating polyneuropathy (CIDP) have been reported [7-11], but in those cases the patients developed their symptoms after $\mathrm{CD}$ had been established during various treatments, making it difficult to determine if they were caused by the disease or were treatment-related. Here, we describe a CIDP-like neuropathy patient who had 3 episodes of motor weakness as the initial presentation of concealed appendiceal $\mathrm{CD}$. The neurological symptoms and abnormal electrophysiologic findings gradually improved after surgical resection of the appendiceal $C D$, without CIDP treatment.

\section{Case presentation}

A 17-year-old male without any medical history was admitted with ascending weakness of both lower extremities that 
had progressed for about two weeks. He had upper respiratory infection one week ago. At the time of admission, his upper respiratory infection was improved and physical examinations in neck, chest and abdomen were unremarkable. On neurologic examination, he had symmetric weakness of both lower extremities (MRC grade 4) with paresthesia of both hands and feet. Deep tendon reflexes were absent in the upper and lower extremities. Routine laboratory findings, including serum complete blood count, biochemistry, urine analysis and C-reactive protein, were normal. Cerebrospinal fluid (CSF) protein level was elevated at $72 \mathrm{mg} / \mathrm{dL}$ without pleocytosis and immunoglobulin G levels in serum $(3370 \mathrm{mg} / \mathrm{dL})$ and CSF $(13.8 \mathrm{mg} / \mathrm{dL})$ were increased. Extensive laboratory investigations including, thyroid function, anti-nuclear antibodies, anti-ganglioside, myelin-associated glycoprotein and viral markers, were all negative. Motor nerve conduction study (NCS) of the median, ulnar, tibial and common peroneal nerves showed prolonged motor terminal latencies and F-wave latencies, and decreased compound muscle action potential (CMAP) amplitudes, sensory nerve action potential (SNAP) amplitude and nerve conduction velocity (NCV) (Table 1). Conduction block and temporal dispersion were also seen in the NCS, compatible with demyelinating polyneuropathy (Figure 1A). With the impression of AIDP, we started intravenous immunoglobulin $(0.4 \mathrm{~g} / \mathrm{kg}$ per day) for 5 days and the patient was discharged after subjective improvement of neurological symptoms.

Eight weeks after the first onset of symptoms, he complained of further progressive motor weakness and tingling sensations in the four extremities. Electrophysiologic studies showed that the demyelinating sensory-motor polyneuropathy had worsened since the previous study (Table 1, Figure 1B). Guillain-Barré syndrome with treatment-related fluctuations or acute-onset CIDP was considered, and

Table 1 Serial nerve conduction study findings

\begin{tabular}{|c|c|c|c|c|}
\hline Nerve & $\begin{array}{l}\text { 1st admission } \\
(2012.5 .15)\end{array}$ & $\begin{array}{l}\text { 2nd admission } \\
\text { (2012.7.19) }\end{array}$ & $\begin{array}{l}\text { 3rd admission } \\
(2012.11 .28)\end{array}$ & $\begin{array}{l}\text { After appendectomy } \\
(2013.6 .18)\end{array}$ \\
\hline \multicolumn{5}{|l|}{ Median motor, right } \\
\hline CMAP amplitude (mV) & 6.1 & 2.5 & 4.6 & 5.1 \\
\hline Distal latency (m/s) & 4.92 & 11.4 & 15.3 & 5.8 \\
\hline $\mathrm{NCV}(\mathrm{m} / \mathrm{s})$ & 32.2 & 16.9 & 14.5 & 20.7 \\
\hline F-wave latency $(\mathrm{m} / \mathrm{s})$ & 40.1 & 33.4 & Absent & 61.9 \\
\hline \multicolumn{5}{|l|}{ Ulnar motor, right } \\
\hline CMAP amplitude (mV) & 9.4 & 6 & 3.8 & 5.3 \\
\hline Distal latency (m/s) & 3.27 & 8.4 & 9.89 & 4.8 \\
\hline $\mathrm{NCV}(\mathrm{m} / \mathrm{s})$ & 32.7 & 21.3 & 12 & 17.8 \\
\hline F-wave latency (m/s) & 33.6 & 41.4 & Absent & 64 \\
\hline \multicolumn{5}{|l|}{ Peroneal motor, right } \\
\hline CMAP amplitude (mV) & 0.62 & No response & No response & 0.37 \\
\hline distal latency $(\mathrm{m} / \mathrm{s})$ & 9.43 & No response & No response & 9.9 \\
\hline $\mathrm{NCV}(\mathrm{m} / \mathrm{s})$ & 27.3 & No response & No response & 17.7 \\
\hline F-wave latency (m/s) & 80.4 & Absent & Absent & Absent \\
\hline \multicolumn{5}{|l|}{ Tibial motor, right } \\
\hline CMAP amplitude (mV) & 3.0 & 0.56 & 0.38 & 2.1 \\
\hline distal latency $(\mathrm{m} / \mathrm{s})$ & 5.63 & 13.6 & 29.9 & 9.1 \\
\hline $\mathrm{NCV}(\mathrm{m} / \mathrm{s})$ & 49.6 & 46.9 & 35.8 & 26.3 \\
\hline F-wave latency (m/s) & 60.2 & 49.3 & Absent & 83.6 \\
\hline \multicolumn{5}{|l|}{ Median sensory, right } \\
\hline SNAP amplitude (mV) & No response & No response & No response & No response \\
\hline $\mathrm{NCV}(\mathrm{m} / \mathrm{s})$ & No response & No response & No response & No response \\
\hline \multicolumn{5}{|l|}{ Sural sensory, right } \\
\hline SNAP amplitude $(\mu \mathrm{V})$ & 17.8 & No response & No response & No response \\
\hline $\mathrm{NCV}(\mathrm{m} / \mathrm{s})$ & 17.9 & No response & No response & No response \\
\hline
\end{tabular}

Abnormal values are shown in bold.

CMAP: compound muscle action potential, SNAP: sensory nerve action potential, NCV: nerve conduction velocity. 


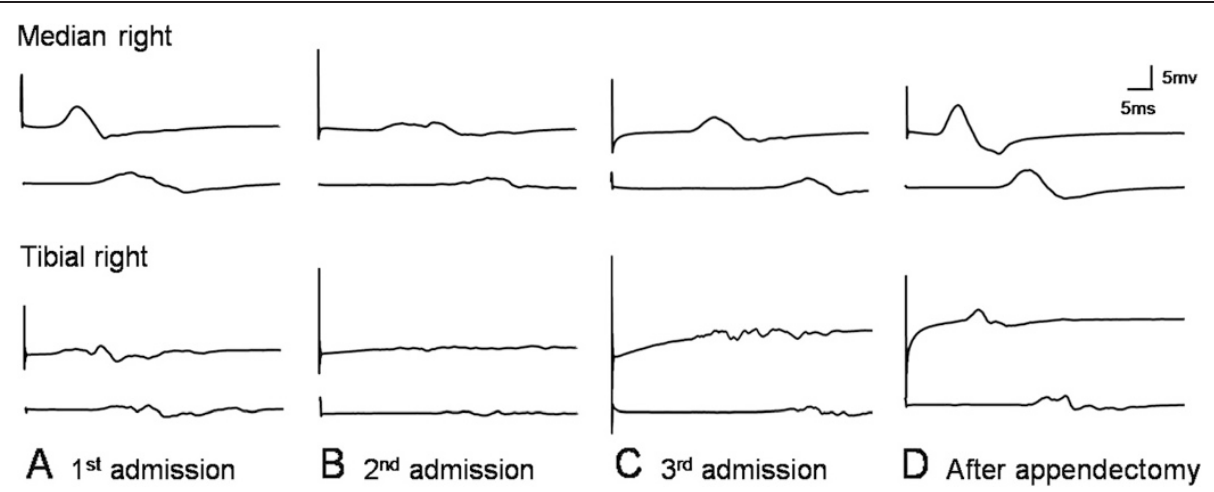

Figure 1 Serial changes of compound motor action potentials (CMAPs) of the median and tibial nerves. (A) The first attack before the first intravenous immunoglobulin, (B) the second attack 8 weeks after the first attack, (C) the third attack 4 months after the second treatment, and (D) 6 months after appendectomy without immuno-modulating therapy.

intravenous immunoglobulin $(0.4 \mathrm{~g} / \mathrm{kg}$ per day $)$ was administered for 5 days, again with clinical improvement of symptoms.

Four months after the second event, the patient complained of recurrent progressive motor weakness and sensory changes. Repeated NCS showed no evoked responses in the median, ulnar, tibial and common peroneal nerves, which was compatible with severe demyelination (Table 1, Figure 1C). With the impression of CIDP, immunoglobulin $(0.4 \mathrm{~g} / \mathrm{kg}$ per day) was administered for 5 days and there was only slight improvement of symptoms.

Four days after the last discharge, the patient visited the emergency department on account of newly developed severe pain in the right lower abdominal quadrant with fever. He had no gastrointestinal symptoms, including diarrhea, prior to development of the abdominal pain. Physical examination of the abdomen revealed tenderness and rebound tenderness on the right lower quadrant. Abdominal computed tomography (CT) prompted suspicion of a periappendiceal abscess, and emergent surgical resection of the ileum, cecum and appendix was performed. Appendiceal biopsy revealed diffuse wall thickening due to transmural lymphocytic infiltration and fibrosis, with scattered non-necrotizing granulomas, which was compatible with Crohn's disease (Figure 2). Since it was localized to the appendix and subsequent gastrofiberscopy and colonofiberscopy were unremarkable, he was diagnosed with appendiceal Crohn's disease. After resection of the appendiceal Crohn's disease his motor weakness and the sensory change in all four limbs gradually recovered without any maintenance treatment. Motor nerve conduction study after seven months showed improvement of electrophysiologic parameters and waveforms (Table 1, Figure 1D). After 12 months of appendectomy, he only showed minimal motor and sensory deficits on extremities and he was able to run. Also there are no gastrointestinal symptoms including diarrhea after surgery until that time.

\section{Conclusions}

We report the first case in which CIDP-like neuropathy was the initial presentation of concealed $C D$. We may assume that the CIDP-like neuropathy was the initial symptom of $\mathrm{CD}$ since recurrent and intractable neurological symptoms preceded the diagnosis of $\mathrm{CD}$, and the
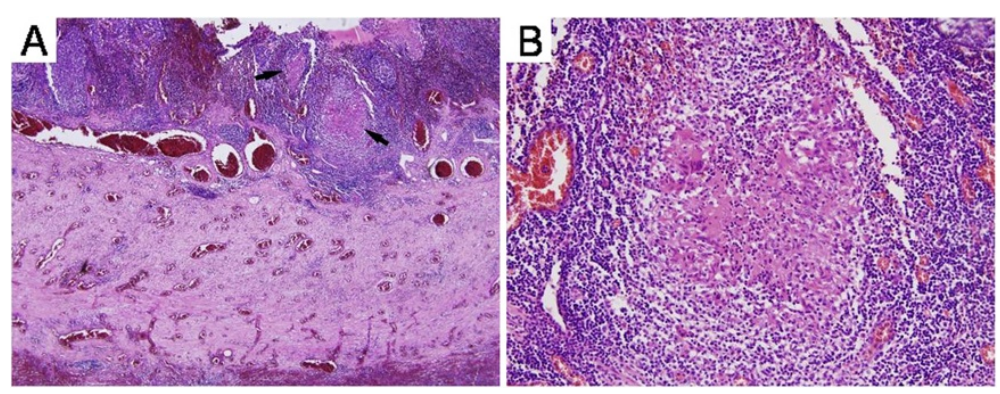

Figure 2 Pathologic findings of Crohn's disease involving the appendix. The appendiceal wall is diffusely thickened by transmural lymphocytic infiltration and fibrosis, with scattered granulomas (A, arrows, H\&E, original magnification x40). A large, well-formed, non-necrotizing granuloma is present in the mucosa $(\mathbf{B}, \mathrm{H} \&$ E, original magnification $\times 200$ ). 
symptoms recovered without maintenance therapy after surgical resection of the appendiceal $\mathrm{CD}$.

The origin of CIDP is still unclear, but it is widely accepted that it is an autoimmune disease with underlying immunopathology involving autoreactive $\mathrm{T}$ cells and B cells [12]. Because CIDP has an autoimmune basis, it can occur in association with diseases such as HIV infection and hepatitis C, Sjogren's syndrome, monoclonal gammopathy of unknown significance, melanoma, lymphoma, diabetes and inflammatory bowel diseases [12]. Only 8 cases of CIDP associated with CD have been reported and we summarized in the Table 2 when clinical information were available [7-11,13]. Of those, 5 cases had already been diagnosed as CD 1 to 30 years before CIDP symptoms developed [7-9]. Since nutritional deficiency or treatment-related neurotoxicity could have occurred during those periods, it is unclear whether the CIDP was caused by the CD itself or by other mechanisms.

In the two other cases the CIDP and CD occurred at the same time, but the patients had only one acute progressive symptom, which was not differentiated from acute inflammatory demyelinating polyradiculoneuropathy (AIDP) $[10,11]$. In addition, they had abdominal symptoms including diarrhea and rectal hemorrhage. Our patient suffered recurrent motor weakness and sensory changes on three occasions over a 7 month period. He also had elevated CSF protein and typical demyelinating-type nerveconduction findings. Although we did not perform a nerve biopsy and did not confirmed same T-cells from appendix, CSF and nerve, we were able to diagnose the patient as CIDP. The most important and unique finding in this case was that his sensory and motor symptoms developed gradually over 7 months, in very close temporal association to the onset of IBD symptoms. Considering that Crohn's disease is not an acute disease with sudden onset, extra-intestinal immunologic effects due to the disease itself may have led to recurrent CIDP like symptoms.
Considering that Crohn's disease is not an acute disease with sudden onset, subclinical immunologic effects due to the disease itself may lead to recurrent CIDP symptoms. Therefore, we would cautiously suggest that we should consider inflammatory bowel disease as a hidden possible concurrent or preceding disease when a patient has recurrent episodes of CIDP.

Another noteworthy finding in our patient is that he recovered from the CIDP symptoms after resection of the appendiceal CD. Although 3 times of IVIG treatment could have influenced the clinical course of the patient, there was only minimal responses before removal of the periappendiceal abscess. Furthermore, the symptoms and electrophysiological findings gradually improved without any treatment for CIDP. Based on our patient's clinical courses, we believe that surgical resection may have more pronounce effect on clinical stabilization of the patient. $\mathrm{CD}$ confined to the appendix occurs in younger patients and is known to be less aggressive and have a low recurrence rate $[14,15]$. It can also be the primary or sole manifestation of the disease. Since, appendiceal biopsy of our patient revealed non-caseous granulomas, and colonofiberscopy showed no granulomatous lesions, he was diagnosed as appendiceal CD. Considering our patient's clinical course, it is possible that the appendiceal $C D$ may have influenced the CIDP-like neuropathy. Appendix is known as an immunological organ, and it may have variable effects on immunologic diseases such as idiopathic inflammatory bowel disease and CIDP-like neuropathy. In this case, CIDP-like neuropathy symptoms were stabilized after surgical resection of the appendiceal $\mathrm{CD}$. We suggest that common immunologic mechanism could be present in those two diseases and non-aggressive limited disease may develop distant neurological symptoms by immunologic mechanisms.

In summary, we describe a case of CIDP as an initial presentation of $\mathrm{CD}$, with recovery of the CIDP symptoms after resection of the appendiceal $\mathrm{CD}$. We suggest

Table 2 Cases of CIDP associated with Crohn's disease

\begin{tabular}{|c|c|c|c|c|c|}
\hline Reference & Age/Sex & IBD activity & Gap & IBD treatment & CIDP treatment \\
\hline our case & $18 / M$ & CA & -7 months & none & IVIG \\
\hline 6 & $60 / F$ & RR & 30 years & TNF-a antagonist (infliximab) & IVIG, prednisolone, azathioprine \\
\hline 7 & $49 / M$ & Q & 20 years & none & $\begin{array}{l}\text { IVIG, plasmapheresis, fludarabine, } \\
\text { methotrexate }\end{array}$ \\
\hline 7 & $47 / F$ & RR & 1 years & etanercept, prednisolone & IVIG, prednisolone, plasmapheresis \\
\hline 7 & $50 / M$ & RR & 23 years & azathioprine & IVIG, prednisolone \\
\hline 8 & $36 / M$ & CA & 4 years & $\begin{array}{l}\text { mesalazine, metronidazole, ciprofloxacin, } \\
\text { azathioprine }\end{array}$ & Plasmapheresis, prednisolone \\
\hline 9 & $32 / \mathrm{M}$ & CA & 0 year & none (newly diagnosed) & IVIG, prednisolone, azathioprine \\
\hline 10 & $63 / F$ & CA & 0 year & none (newly diagnosed) & IVIG, prednisolone \\
\hline
\end{tabular}

$\mathrm{CA}$, currently active; $\mathrm{Q}$, quiescent; $\mathrm{RR}$, remitting/relapsing; $\mathrm{UK}$, unkown; IVIg, intravenous immunoglobulin. 
that inflammatory bowel disease should be considered a possible underlying disease when investigating patients with CIDP.

\section{Consent}

Written informed consent was obtained from the patient for publication of this Case report and any accompanying images. A copy of the written consent is available for review by the Editor of this journal.

\section{Abbreviations}

CIDP: Chronic inflammatory demyelinating polyneuropathy; CD: Crohn's disease; IVIG: Intravenous immunoglobulin; CSF: Cerebrospinal fluid: NCS: Nerve conduction study; CMAP: Compound muscle action potential; SNAP: Sensory nerve action potential; NCV: Nerve conduction velocity; AIDP: Acute inflammatory demyelinating polyradiculoneuropathy.

\section{Competing interests}

The authors declare that they have no competing interests.

\section{Authors' contribution}

SK contributed to draft the manuscript. SJK contributed to data acquisition and analysis of publication. KWO have made contribution to acquisition of data and analysis and interpretation of data. BKA, HLL, and KJ contributed to data acquisition. DSH conceived study design and had made contributions to analysis and interpretation of data. YSK performed conceived of the study, data acquisition, analysis and interpretation of data, and contributed to draft the manuscript. All authors read and approved the final manuscript.

\section{Acknowledgment}

The authors would like to thank their patient for participation in this study. This work was supported by the research fund of Hanyang University (HY-2013).

\section{Author details}

${ }^{1}$ Department of Radiology, Kangbuk Samsung Medical Center, Seoul, Republic of Korea. ${ }^{2}$ Department of Neurology, College of Medicine, Hanyang University,

17 Haengdang-dong, Seongdong-gu, Seoul 133-792, Republic of Korea. ${ }^{3}$ Department of General Surgery, College of Medicine, Hanyang University, Seoul, Republic of Korea. ${ }^{4}$ Department of Gastroenterology, College of Medicine, Hanyang University, Seoul, Republic of Korea. ${ }^{5}$ Department of Pathology, College of Medicine, Hanyang University, Seoul, Republic of Korea.

Received: 15 September 2014 Accepted: 11 March 2015

Published online: 28 March 2015

\section{References}

1. Baumgart DC, Sandborn WJ. Inflammatory bowel disease: clinical aspects and established and evolving therapies. Lancet. 2007;369:1641-57.

2. Lossos A, River Y, Eliakim A, Steiner I. Neurologic aspects of inflammatory bowel disease. Neurology. 1995;45(3 Pt 1):416-21.

3. Benavente L, Morís $G$. Neurologic disorders associated with inflammatory bowel disease. Eur J Neurol. 2011:18:138-43.

4. Ferro JM, Oliveira S. Neurologic manifestations of gastrointestinal and liver diseases. Curr Neurol Neurosci Rep. 2014;14:487.

5. Sassi SB, Kallel L, Ben Romdhane S, Boubaker J, Filali A, Hentati F. Peripheral neuropathy in inflammatory bowel disease patients: a prospective cohort study. Scand J Gastroenterol. 2009;44:1268-9.

6. Figueroa JJ, Loftus EV, Harmsen WS, Dyck PJB, Klein CJ. Peripheral neuropathy incidence in inflammatory bowel disease: a population-based study. Neurology. 2013;80:1693-7.

7. Lozeron P, Denier C, Lacroix C, Adams D. Long-term course of demyelinating neuropathies occurring during tumor necrosis factor-alpha-blocker therapy. Arch Neurol. 2009:66:490-7.

8. Gondim FAA, Brannagan TH, Sander HW, Chin RL, Latov N. Peripheral neuropathy in patients with inflammatory bowel disease. Brain. 2005;128(Pt 4):867-79.

9. Ohyagi M, Ohkubo T, Yagi Y, Ishibashi S, Akiyama J, Nagahori M, et al. Chronic Inflammatory Demyelinating Polyradiculoneuropathy in a Patient with Crohn's Disease. Intern Med. 2013;52:125-8.
10. Boylu E, Toğrol E, Doğan T, Saraçoğlu M. Crohn disease and chronic inflammatory demyelinating polyneuropathy; a case report. Electromyogr Clin Neurophysiol. 2010;50:181-5.

11. Ariatti A, Ficarra G, Girolami F, Pentore R, Galassi G. Chronic inflammatory demyelinating polyradiculoneuropathy associated with inflammatory bowel diseases: questioning the autoimmunity hypothesis. Int J Colorectal Dis. 2009;24:603-4.

12. Köller H, Kieseier BC, Jander S, Hartung H-P. Chronic inflammatory demyelinating polyneuropathy. N Engl J Med. 2005;352:1343-56.

13. Oliveira GR, Teles BCV, Brasil EF, Souza MHLP, Furtado LET, De Castro-Costa CM, et al. Peripheral neuropathy and neurological disorders in an unselected Brazilian population-based cohort of IBD patients. Inflamm Bowel Dis. 2008;14:389-95.

14. Bischoff A, Gupta A, D'Mello S, Mezoff A, Podberesky D, Barnett S, et al, Crohn's disease limited to the appendix: a case report in a pediatric patient. Pediatr Surg Int. 2010;26:1125-8.

15. Prieto-Nieto I, Perez-Robledo JP, Hardisson D, Rodriguez-Montes JA, Larrauri-Martinez J, Garcia-Sancho-Martin L. Crohn's disease limited to the appendix. Am J Surg. 2001;182:531-3.

\section{Submit your next manuscript to BioMed Central and take full advantage of:}

- Convenient online submission

- Thorough peer review

- No space constraints or color figure charges

- Immediate publication on acceptance

- Inclusion in PubMed, CAS, Scopus and Google Scholar

- Research which is freely available for redistribution 